\title{
La diversificación del comercio de Nueva Zelanda: el caso de México
}

\author{
José Jaime López Jiménez*
}

\section{Introducción}

$\mathrm{N}$

ueva Zelanda es actualmente una de las tres economías, junto con Japón y Australia, consideradas como desarrolladas en el Pacífico asiático. Si bien es, con mucho, la más pequeña de las tres, puesto que apenas representa poco más de 1 por ciento de la economía japonesa, sobresale en el comercio internacional por el alto grado de apertura de su economía como resultado de las reformas económicas implementadas durante la década de los años ochenta, para ubicarse entre las 10 principales economías de mayor orientación al exterior a nivel mundial. ${ }^{1}$ En el ámbito internacional forma parte de diversos foros de cooperación regional, entre ellos el Foro de Cooperación Económica Asia-Pacifico (APEC, por sus siglas en inglés) y la Asociación de Naciones del Sureste de Asia (ASEAN, por sus siglas en inglés), así como de otros organismos internacionales como la Organización Mundial de Comercio (OMC) y la Organización para la Cooperación y el Desarrollo Económico (OCDE).

Desde la década de los años setenta este pequeño país emprendió una nueva estrategia comercial orientada a diversificar tanto sus mercados como la base de sus exportaciones, ante las exigencias que

* Profesor-investigador del Departamento de Estudios del Pacífico de la Universidad de Guadalajara, e investigador del sNI. la nueva realidad le impuso en esos años, pero también en un intento por ampliar su presencia en el mercado mundial. En este contexto, México podría convertirse en un mercado estratégico para Nueva Zelanda, en su afán de ampliar sus relaciones comerciales en el continente americano.

El propósito principal de este artículo es analizar la relación bilateral actual entre Nueva Zelanda y México, para evaluar el potencial de dicha relación. En una primera sección se presenta de manera resumida, como marco de referencia general, la evolución de las relaciones comerciales de Nueva Zelanda con el exterior. En la segunda sección se analiza la relación comercial entre los dos países. Finalmente, se presentan las conclusiones del trabajo.

\section{Los primeros contactos de Nueva Zelanda con el exterior}

Los primeros pobladores de Nueva Zelanda fueron los maorí, aborígenes de la raza polinesia que llegaron al país hace alrededor de mil años, procedentes básicamente de las Islas Cook en oleadas sucesivas, la última de ellas ocurrida en el siglo XIV. Algunos documentos sobre la historia de este país señalan que fue el navegante polinesio Kupe quien descubrió este archipiélago y lo nombró Aotearoa, que significa Tierra de la Larga Nube Blanca, denominación que sigue siendo el nombre maorí de Nueva Zelanda. En el tiempo en que arribaron 
los primeros exploradores europeos la población oscilaba entre 100,000 y 200,000 habitantes. ${ }^{2}$

El primer europeo en arribar fue el navegante holandés Abel Janszoon Tasman en 1642, quien descubrió las islas de la hoy Nueva Zelanda en uno de sus viajes de exploración de las costas de la también recientemente descubierta Australia, nombrando a las nuevas islas como Nieuw Zeeland, en honor de una provincia holandesa, pero sin llegar a establecer ningún vínculo de importancia con las nuevas tierras. Posteriormente, durante el siglo XVIII Inglaterra envió varias expediciones al Pacífico Sur, siendo así como en 1769 llegó el explorador inglés James Cook en el primero de tres viajes que realizó a Nieuw Zeeland y estableció los primeros contactos formales con los maoríes, sentando con ello las bases para el asentamiento de europeos en territorio neozelandés. A partir de 1790 se comenzaron a establecer campamentos que servirían como bases costeras de operaciones y en 1792 se fundó el primer asentamiento británico en Russell, en la Isla Norte, en lo que se denominó como Bahía de las Islas.

Pese al contacto con los extranjeros, la cultura maorí permaneció intacta casi hasta principios del siglo XIX, cuando los mapas y referencias de Cook sobre las nuevas islas atrajeron numerosos cazadores de ballenas y focas por la abundancia de estos mamíferos en sus aguas; así como traficantes de lino, madera, frutos y mujeres, que inter- cambiaban por licores, armas y diversas mercancías procedentes de Europa. De esta forma, los maoríes entraron en contacto con una civilización material superior, acelerándose este proceso con la llegada de misioneros, quienes introdujeron nuevos conceptos espirituales que transformaron paulatinamente a la sociedad maorí.

En 1840 se había formado una numerosa colonia de más de 2,000 pobladores europeos, en su mayoría cazadores marinos y comerciantes, así como renegados que habían huido de sus barcos y ex convictos de las colonias penales de Australia, a quienes muchos maoríes veían como intrusos blancos y deseaban expulsarlos, pero otros veían en ellos la oportunidad de conocer sus técnicas de trabajo y de establecer un intercambio comercial con Europa. Este contacto con los europeos tuvo dos vertientes: por una parte, se introdujeron nuevos aperos de labranza en la agricultura, ganado bovino y ovino, y maquinaria para la industria y la minería, dándole un fuerte impulso a la economía del país; por otra, el licor y las enfermedades introducidas por los inmigrantes causaron grandes estragos, mientras que el uso del fusil en las guerras tribales maoríes diezmó significativamente a la población nativa.

Por otro lado, a partir de 1840 las relaciones entre maoríes y británicos quedarían formalizadas mediante la firma del Tratado de Waitangi entre la Corona — por conducto del capitán William Hobson-y los jefes 
maoríes. Con este tratado, los nativos cedieron a los ingleses el derecho de comprar tierra maorí a cambio de derechos y privilegios como súbditos del Reino Unido, pero con una cláusula que les respetaba los derechos de propiedad sobre sus tierras, bosques y recursos pesqueros. De esta forma, Nueva Zelanda, que hasta entonces había fungido como parte de la colonia penitenciaria de Nueva Gales del Sur, hoy Australia, se convirtió rápidamente en una colonia británica más. ${ }^{3}$

Dos décadas más tarde, en 1861, dos sucesos permitieron un rápido desarrollo de Nueva Zelanda: el descubrimiento de yacimientos de oro y el fin de las guerras entre los maoríes. La fiebre del oro abrió la puerta a una colonización masiva de inmigrantes europeos, principalmente ingleses, lo que a su vez dio un fuerte impulso a la agricultura y la ganadería. Este suceso, que se produjo en la Isla Sur, impulsó al gobierno a establecer su sede en un punto más central y estratégico, eligiéndose a Wellington como capital del país en 1865, en el extremo sur de la Isla Norte. Posteriormente, el descubrimiento también de oro en la Isla Norte transformaría el país entero, impulsándose el rápido desarrollo de los ferrocarriles, puertos, carreteras y construcciones de todo tipo, lo que facilitó el movimiento de personas y mercancías tanto al interior como al exterior del país.

Todo ello propició una relación comercial mucho más amplia con los británicos, a quienes exportaba principalmente carne, lana y productos lácteos y de quienes importaba manufacturas, combustibles e insumos para la industria local. No obstante, en 1879 el gobierno de Nueva Zelanda enfrentó la primer crisis económica de su historia derivada en gran medida del intercambio desigual que sostenía con los ingleses, intercambio que se reducía básicamente a la venta de materias primas y compra de productos manufacturados, que empujó al país a tomar una serie de medidas proteccionistas con la intención de estimular el crecimiento de la producción nacional. Pero esto sólo provocó que Nueva Zelanda se volviera más dependiente de Gran Bretaña, ya que esta última se convirtió rápidamente en el principal mercado de sus exportaciones y en su mayor fuente de capital.

Bajo estas circunstancias, a principios del siglo xx el Partido Laboral ascendió al poder y favoreció la expansión de la economía agrícola mediante la organización y regulación de los mercados, el impulso a las organizaciones de productores, la creación de esquemas de crédito rural y la construcción de infraestructura rural, entre otras acciones. Por otra parte, el hecho de contar con una población relativamente pequeña y con una población rural que constituía 30 por ciento de la población total, favoreció el carácter extensivo de las explotaciones agropecuarias y una rápida mecanización de la producción, en donde la mayor parte de las familias rurales se hizo de propiedades con vastas extensiones orientadas a la ganadería, en virtud de la gran disponibilidad de pastizales naturales y de que esta actividad requería de menos mano de obra que la agricultura.

No obstante, a principios de los años setenta se presentaron dos sucesos que marcarían un nuevo rumbo en el desarrollo económico y en las relaciones comerciales de Nueva Zelanda con el exterior. Por un lado, la crisis mundial del petróleo de 1973 provocó el colapso de los precios internacionales de las mercancías en general, afectando sensiblemente el sector más importante para la economía neozelandesa: el sector exportador. De un crecimiento de 17 por ciento anual observado entre 1967 y 1973 , las exportaciones fueron disminuyendo 
hasta quedar prácticamente estancadas a comienzos de los años ochenta.

Por otro lado, en 1974 Gran Bretaña se incorporó a la Comunidad Económica Europea, lo que en la práctica representó para Nueva Zelanda perder a su principal socio comercial hasta ese año, en virtud de que ese país constituía el destino de aproximadamente 80 por ciento de sus exportaciones y el origen de 50 por ciento de las importaciones. ${ }^{4}$ Ante esta circunstancia, el gobierno de Nueva Zelanda emprendió una nueva política comercial en función de un doble objetivo. Por un lado, se buscó diversificar los países de destino de sus exportaciones y, por otro, ampliar la oferta de bienes para mantener su participación en el mercado internacional.

\section{La diversificación de mercados y la apertura comercial}

La nueva estrategia de diversificación del comercio se orientó principalmente hacia los países del Pacífico, en donde destacan Australia, Estados Unidos, Japón, China y Corea del Sur como sus principales socios comerciales, además de Gran Bretaña, en tanto que sus tradicionales exportaciones agropecuarias de carne, lana y productos lácteos, paulatinamente se fueron extendiendo a productos hortofrutícolas, forestales, pesqueros y manufacturas. Esta estrategia se apoyó fundamentalmente en el subsidio a las exportaciones y en una liberalización parcial de las importaciones iniciada en 1978, la cual serviría como antecedente para la posterior liberalización de la economía en general.

Entre las principales medidas que se adoptaron sobresalía la sustitución gradual de licencias de importación por aranceles, mismos que irían disminuyendo en forma progresiva. Es importante anotar que este proceso de liberalización del comercio se llevó a cabo de manera unilateral; empero, también es conveniente destacar que simultáneamente se aplicaron diversos mecanismos de fomento a la producción interna, por ejemplo planes de desarrollo industrial acordes con los plazos de sustitución de licencias de importación por aranceles y con la posterior desgravación arancelaria; de igual forma, se aplicaron planes de estabilización de precios para proteger el ingreso de los agricultores ante las fluctuaciones de los precios internacionales.

Como parte de la nueva política comercial adoptada con las reformas económicas en 1984, el gobierno de Nueva Zelanda instauró una atmósfera empresarial de competencia internacional. Para ello, se continuó con la reducción unilateral de los aranceles, los cuales disminuyeron de un máximo de 60 por ciento, hasta 20 por ciento, y se eliminó la mayor parte de las licencias de importación; sólo en aquellos productos que no estaban contemplados en los planes industriales se estableció un arancel de 25 por ciento. A partir de 1987 y hasta mediados de los años noventa, los aranceles fueron disminuyendo gradualmente a fin de mantenerlos en 10 por ciento para todos los sectores, excepto en algunos rubros como calzado, alfombras, ropa y vehículos. ${ }^{5}$ En el sector agropecuario, en 2001 Nueva Zelanda removió las tarifas en bienes procedentes de países menos desarrollados, excepto en productos de papel, las cuales se eliminaron finalmente a partir de $2004 .^{6}$

En la actualidad no sólo se han eliminado todas las restricciones cuantitativas y sustituidas por aranceles, sino que estos últimos prácticamente se han reducido a cero, procurando una mayor neutralidad de la política comercial hacia todos los sectores productivos. No obstante lo anterior, al igual que en la mayoría de los países desarrolla- 
dos, continúan aplicándose restricciones no arancelarias a las importaciones por razones ambientales, fitosanitarias, de seguridad, de protección de derechos de propiedad intelectual, así como otro tipo de sanciones convenidas por la comunidad internacional y normas claras de especificación de los productos.

Por otro lado, como parte de la política de diversificación de las relaciones comerciales con el exterior, en 1983 se firmó el Acuerdo Comercial de Relaciones Económicas más Estrechas entre Australia-Nueva Zelanda (ANZCERTA, por sus siglas en inglés); al mismo tiempo se apoyaron las negociaciones multilaterales para la liberalización del comercio al interior de la omc y los acuerdos regionales con este mismo fin, particularmente los relativos al APEC. No obstante, ante los pocos avances en las negociaciones multilaterales en los últimos años Nueva Zelanda ha optado por concesiones comerciales recíprocas mediante acuerdos regionales y bilaterales de libre comercio. Bajo este nuevo enfoque, además de ampliar y fortalecer los términos del ANZCERTA, Nueva Zelanda firmó recientemente un acuerdo comercial con Singapur e inició negociaciones con este mismo fin con Hong Kong y China, negocia otro acuerdo de manera conjunta con Chile y Singapur y está estudiando la posibilidad de establecer acuerdos comerciales con México y Corea del Sur.

\section{El comercio de Nueva Zelanda}

Para el año 2005 las exportaciones de bienes de Nueva Zelanda alcanzaron $22 \mathrm{mil}$ millones de dólares, en tanto que las importaciones ascendieron a 24 mil millones de dólares, que constituyen 21 y 22 por ciento del PIB, respectivamente. ${ }^{7}$ De igual forma, las exportaciones de bienes representaron en ese mismo año alrededor de 72 por ciento de las exportaciones totales, en tanto que las importaciones de bienes alcanzaron 76 por ciento del total de importaciones. Ahora bien, dentro de las exportaciones de bienes alrededor de 62 por ciento provienen del sector agropecuario, incluyendo productos forestales; 32 por ciento son manufacturas, 4 por ciento productos pesqueros, y el resto corresponde a minerales y químicos. Tomando en cuenta que se trata de un país desarrollado, es importante destacar el hecho de que Nueva Zelanda mantiene una fuerte dependencia de las exportaciones agropecuarias, situación que contrasta con la gran mayoría de las economías desarrolladas, en las cuales la agricultura generalmente presenta una baja participación en el sector externo, con un promedio de 8 por ciento del total de exportaciones. ${ }^{8}$

Esta alta dependencia de Nueva Zelanda de las exportaciones agropecuarias ha traído como consecuencia una serie de inconvenientes para su economía. En primer lugar, buena parte de los productos agrícolas enfrenta rígidos esquemas proteccionistas en los países desarrollados, particularmente en Estados Unidos y Japón — dos de los principales socios comerciales de Nueva Zelanda-, situación que implica un verdadero freno y evita un mayor dinamismo del sector exportador neozelandés. En segundo lugar, los bajos precios relativos de los productos agrícolas en el mercado internacional han impactado de forma adversa las exportaciones del sector, no obstante la ventaja comparativa existente en la agricultura del país, lo que ha traído como consecuencia negativos términos de intercambio para Nueva Zelanda durante los últimos años.

En cuanto a las importaciones de bienes, 80 por ciento del total corresponde a manufacturas, 12 por ciento a combustibles y el 8 por ciento restante a productos 
La diversificación del comercio de Nueva Zelanda: el caso de México

Cuadro 1

Nueva Zelanda: Comercio exterior de bienes, 2000-2005

(porcentajes)

\begin{tabular}{lrrrrrr}
\hline Exportaciones & 2000 & 2001 & 2002 & 2003 & 2004 & 2005 \\
\hline Total & 100.0 & 100.0 & 100.0 & 100.0 & 100.0 & 100.0 \\
Productos agropecuarios & 50.3 & 54.4 & 52.4 & 52.1 & 54.0 & 52.9 \\
$\quad$ Productos lácteos & 19.8 & 24.0 & 20.6 & 19.9 & 20.3 & 18.8 \\
Carne y productos de carne & 13.5 & 14.0 & 14.6 & 14.8 & 15.7 & 15.7 \\
Frutas y vegetales & 5.6 & 5.0 & 5.5 & 5.5 & 6.1 & 5.7 \\
Lana & 3.4 & 2.9 & 3.1 & 3.2 & 3.0 & 2.7 \\
Cueros y pieles & 2.3 & 2.6 & 2.3 & 2.2 & 1.8 & 1.5 \\
Otros productos agrícolas & 5.7 & 5.9 & 6.3 & 6.6 & 7.1 & 8.5 \\
Productos forestales & 12.2 & 10.9 & 11.0 & 11.0 & 9.9 & 9.4 \\
Pescado & 4.8 & 4.5 & 4.8 & 4.5 & 4.1 & 4.0 \\
Manufacturas & 27.5 & 26.0 & 29.9 & 30.3 & 30.6 & 32.0 \\
$\quad$ Maquinaria & 7.8 & 7.4 & 7.5 & 7.8 & 8.1 & 8.6 \\
Metal y artículos de metal & 6.9 & 6.2 & 6.1 & 6.1 & 6.1 & 6.6 \\
Textil, vestido y calzado & 1.1 & 1.1 & 1.1 & 1.2 & 1.3 & 1.3 \\
Otras manufacturas & 11.7 & 11.3 & 15.2 & 15.1 & 15.2 & 15.6 \\
Combustibles minerales y químicos & 5.2 & 4.3 & 1.9 & 2.1 & 1.4 & 1.7 \\
\hline Importaciones & 2000 & 2001 & 2002 & 2003 & 2004 & 2005 \\
\hline Total & 100.0 & 100.0 & 100.0 & 100.0 & 100.0 & 100.0 \\
Vehículos & 12.3 & 11.5 & 13.8 & 15.5 & 16.0 & 15.3 \\
Maquinaria & 13.0 & 12.9 & 13.7 & 13.5 & 13.6 & 13.7 \\
Combustibles minerales & 7.9 & 11.2 & 9.0 & 9.8 & 9.4 & 11.6 \\
Maquinaria eléctrica & 10.1 & 10.6 & 8.8 & 8.4 & 9.3 & 9.0 \\
Plásticos & 4.1 & 4.2 & 4.1 & 4.0 & 3.7 & 3.9 \\
Material fotográfico & 2.9 & 3.1 & 3.1 & 3.0 & 3.1 & 3.1 \\
Papel & 2.9 & 2.9 & 2.9 & 2.9 & 2.8 & 2.6 \\
Fármacos & 2.5 & 2.5 & 2.4 & 2.3 & 2.4 & 2.5 \\
Aeronaves & 5.2 & 2.8 & 2.6 & 2.5 & 3.4 & 2.0 \\
Artículos de hierro y de acero & 1.4 & 1.4 & 1.5 & 1.6 & 1.6 & 1.9 \\
Otros productos & 37.7 & 36.9 & 38.1 & 36.5 & 34.7 & 34.4 \\
\hline Fer & & & &
\end{tabular}

Fuente: Ministry of Foreign Affairs and Trade, External Trade Statistics, Nueva Zelanda, 2005.

agrícolas. Los principales bienes manufacturados importados son vehículos y maquinaria, con cerca de 30 por ciento del total; le siguen combustibles con alrededor de 12 por ciento; maquinaria eléctrica 9 por ciento; plásticos 4 por ciento, y material fotográfico 3 por ciento (véase cuadro 1).

Por otra parte, las exportaciones de servicios constituyen otro rubro importante del comercio exterior de Nueva Zelanda, en virtud del rápido crecimiento de ese sector en los últimos años. Cabe señalar que en el año 2005 los servicios representaron 28 por ciento del total de exportaciones del país, mientras que las importaciones de servicios alcanzaron 24 por ciento del total. ${ }^{9}$ Entre los servicios que Nueva Zelanda exporta sobresalen los viajes personales y de negocios y los transportes, mismos que representan 64 y 19 por ciento, respectivamente, del total de las exportaciones de servicios. Del lado de las importaciones de 
Cuadro 2

Nueva Zelanda: Comercio exterior de servicios, 2000-2005 (porcentajes)

\begin{tabular}{lrrrrrr}
\hline & 2000 & 2001 & 2002 & 2003 & 2004 & 2005 \\
\hline Exportación total de servicios & 100.0 & 100.0 & 100.0 & 100.0 & 100.0 & 100.0 \\
Viajes personales y de negocios & 52.1 & 50.5 & 56.0 & 59.1 & 62.9 & 64.2 \\
Transportes & 28.8 & 28.1 & 24.4 & 21.9 & 20.1 & 18.9 \\
Comunicaciones & 4.3 & 4.1 & 3.8 & 3.2 & 2.7 & 2.3 \\
Computación e información & 1.6 & 1.9 & 1.7 & 1.8 & 1.5 & 1.6 \\
Cultura y recreación & 2.5 & 3.2 & 2.2 & 1.3 & 1.1 & 1.8 \\
Licencias y derechos de autor & 1.2 & 1.2 & 1.1 & 1.9 & 1.8 & 1.2 \\
Servicios gubernamentales & 1.3 & 1.1 & 1.3 & 1.2 & 1.2 & 1.2 \\
Otros servicios & 8.2 & 9.9 & 9.5 & 9.6 & 8.7 & 8.8 \\
\hline Importación total de servicios & 100.0 & 100.0 & 100.0 & 100.0 & 100.0 & 100.0 \\
\hline Transportes & 32.1 & 31.4 & 34.6 & 35.4 & 35.1 & 36.3 \\
Viajes personales y de negocios & 33.1 & 33.0 & 31.1 & 31.2 & 32.2 & 34.3 \\
Licencias y derechos de autor & 6.9 & 7.1 & 7.3 & 7.4 & 7.7 & 6.9 \\
Comunicaciones & 4.1 & 4.4 & 3.0 & 3.3 & 2.9 & 2.9 \\
Seguros & 2.4 & 2.2 & 2.5 & 2.8 & 3.0 & 2.9 \\
Computación e información & 2.3 & 2.2 & 2.1 & 2.2 & 1.6 & 2.2 \\
Servicios gubernamentales & 1.5 & 1.3 & 1.5 & 1.6 & 1.6 & 1.3 \\
Otros servicios & 17.5 & 18.4 & 17.9 & 16.1 & 15.9 & 13.2 \\
\hline
\end{tabular}

Fuente: Ministry of Foreign Affairs and Trade, External Trade Statistics, Nueva Zelanda, 2005.

servicios, también sobresalen los rubros de transportes y de viajes personales y de negocios, que en conjunto representan alrededor de 70 por ciento del total de importaciones de servicios que realiza el país, lo que refleja la trascendencia del flujo de bienes y de personas hacia y desde el exterior para Nueva Zelanda (cuadro 2).

Por otro lado, a partir de la diversificación de las relaciones comerciales con el exterior que el país emprendió desde los años setenta, Nueva Zelanda experimentó un importante cambio en sus vínculos con los países del Pacífico. De tal suerte que, si antes concentraba su comercio con Europa -particularmente con Gran Bretaña- de manera similar como México lo hace actualmente con Estados Unidos, en los últimos años se ha observado una reorientación de sus vínculos comerciales principalmente hacia Australia, Estados Unidos y Japón. Cabe mencionar que en 1990 Australia había desplazado a la Unión Europea como el principal socio comercial de Nueva Zelanda, y para 2005 absorbía ya 20 por ciento de las exportaciones y aportaba 22 por ciento de las importaciones neozelandesas; en segundo lugar, y con una participación descendente, se ubicó la Unión Europea con 16 y 19 por ciento, respectivamente, siguiéndole de cerca Estados Unidos y Japón. Cabe destacar aquí el notable crecimiento que ha registrado el comercio de Nueva Zelanda con China en los últimos años, país con el cual el intercambio aumentó de niveles de 1 por ciento ciento tanto en las exportaciones como en las importaciones, a alrededor de 


\section{Cuadro 3}

Nueva Zelanda: Cambios en las relaciones comerciales (Estructura porcentual)

\begin{tabular}{lrrrrrrrr}
\hline País & \multicolumn{4}{c}{ Exportaciones } \\
\hline & 1978 & 1990 & 2000 & 2005 & 1978 & 1990 & 2000 & 2005 \\
\hline Total & 100.0 & 100.0 & 100.0 & 100.0 & 100.0 & 100.0 & 100.0 & 100.0 \\
Unión Europea* & 30.4 & 16.4 & 14.3 & 16.1 & 27.3 & 25.0 & 18.4 & 19.4 \\
$\quad$ Gran Bretaña & 18.1 & 7.1 & 5.2 & 4.7 & 15.9 & 7.4 & 4.4 & 3.3 \\
Australia & 10.9 & 18.2 & 19.3 & 20.1 & 20.7 & 20.3 & 22.2 & 22.2 \\
Estados Unidos & 14.0 & 13.1 & 14.2 & 14.2 & 13.8 & 17.9 & 17.4 & 10.2 \\
Japón & 13.8 & 15.7 & 13.2 & 11.7 & 13.9 & 15.5 & 11.3 & 11.0 \\
China & 1.6 & 1.0 & 2.8 & 5.3 & 0.6 & 0.7 & 6.2 & 10.3 \\
Corea del Sur & 1.7 & 4.2 & 4.2 & 3.7 & 2.8 & 1.6 & 2.2 & 2.8 \\
Otros países & 27.6 & 31.4 & 32.0 & 28.9 & 20.9 & 19.0 & 22.3 & 24.1 \\
\hline
\end{tabular}

* Incluyendo a Gran Bretaña

Fuente: CEPAL, El proceso exportador en tres agriculturas templadas del Hemisferio Sur (Australia, Nueva Zelanda y Sudáfrica), LC/R. 1350, 30 de diciembre de 1993; International Monetary Found, Direction of Trade Statistics, 2001; Ministry of Foreign Affairs and Trade, External Trade Statistics, Nueva Zelanda, 2005.

5 y 10 por ciento, respectivamente, en poco más de una década, lo que da una idea del fuerte crecimiento en la relación comercial entre ambas naciones (cuadro 3).

Sin duda alguna, en términos generales las relaciones económicas de Nueva Zelanda con los países del Pacífico han cobrado una gran importancia en los últimos años. A fines de los años setenta, Australia, Estados Unidos, Japón, China y Corea del Sur concentraban en conjunto 42 por ciento de las exportaciones y 52 por ciento de las importaciones neozelandesas, en tanto que a mediados de la presente década el comercio con esos mismos países aumentó a 55 por ciento en el caso de las primeras y a 56 por ciento en las segundas, lo que denota un mayor grado de integración con la región del Pacífico Asiático (cuadro 3).

\section{La relación comercial entre Nueva Zelanda y México}

Tradicionalmente la relación comercial entre Nueva Zelanda y México ha sido poco significativa para ambos; en mayor medida para el último. No obstante, en la última década el comercio bilateral entre los dos países registró un notable crecimiento al pasar de 135 a 361 millones de dólares, esto es, un incremento medio anual de 10.3 por ciento. Este rápido crecimiento se puede apreciar más claramente en las exportaciones de México hacia Nueva Zelanda, las cuales pasaron de 3 a 84 millones de dólares en diez años, aunque representan una mínima participación en las importaciones neozelandesas. De igual forma, aunque en menor proporción que las importaciones, las exportaciones de Nueva Zelanda hacia México mostraron un aumento significativo al duplicarse durante la misma década, y presentan una participaron de 1.4 por ciento del total de las exportaciones neozelandesas (cuadro 4). Esta situación en cierta forma refleja la prioridad que los dos países han otorgado a sus relaciones económicas con otras regiones: Nueva Zelanda con los países del Pacífico Asiático y México con Norteamérica. 
$\underline{\text { Análisis }}$

Cuadro 4

Nueva Zelanda: Comercio con México (millones de dólares)

\begin{tabular}{ccccc}
\hline Año & $\begin{array}{c}\text { Exportaciones } \\
\text { a México }\end{array}$ & $\%$ & $\begin{array}{c}\text { Importaciones } \\
\text { de México }\end{array}$ & $\%$ \\
\hline 1996 & 132 & 0.1 & 3 & 0.0 \\
1997 & 151 & 0.1 & 4 & 0.0 \\
1998 & 156 & 0.1 & 7 & 0.0 \\
1999 & 147 & 0.1 & 9 & 0.0 \\
2000 & 126 & 0.9 & 72 & 0.5 \\
2005 & 277 & 1.4 & 84 & 0.4 \\
\hline
\end{tabular}

Fuente: Bancomext, Estadísticas de Comercio Exterior. Ministry of Foreign Affairs and Trade, External Trade Statistics, Nueva Zelanda, 2005.

Cabe mencionar que la estructura del comercio en los dos países ha registrado cambios importantes en las últimas décadas. En el caso de Nueva Zelanda, si bien los bienes tradicionales como los lácteos, la carne y la lana aún mantienen una alta participación en las exportaciones del país, paulatinamente se han ido incorporando otros bienes como frutas, hortalizas, productos forestales y pesqueros, así como manufacturas diversas. En el caso de México, la transformación de su sector exportador ha sido mucho más evidente, puesto que durante la década de los años ochenta las exportaciones se centraban básicamente en petróleo y algunos productos agrícolas. Por el contrario, para la década de los noventa esos bienes redujeron su participación en el sector exportador ante el auge de las manufacturas, mismas que han llegado a representar casi tres cuartas partes de las exportaciones totales de México. ${ }^{10}$ En el caso de las importaciones, en ambos países éstas se integran en su mayor parte por bienes manufacturados.

En relación con el intercambio bilateral, se puede resaltar el hecho de que Nueva Zelanda ha inclinado la balanza comercial a su favor en términos absolutos, lo que se ha traducido en un creciente déficit para México, aunado al hecho de que las

\section{Cuadro 5}

Nueva Zelanda: exportaciones hacia México, 1996-2005 (estructura porcentual)

\begin{tabular}{lrrrrrr}
\hline Productos & 1996 & \multicolumn{1}{c}{1997} & 1998 & 1999 & 2000 & 2005 \\
\hline Total & 100.0 & 100.0 & 100.0 & 100.0 & 100.0 & 100.0 \\
Agropecuarias & 87.0 & 87.5 & 84.0 & 87.5 & 79.7 & 85.5 \\
$\quad$ Lácteos & 81.3 & 80.4 & 77.3 & 79.5 & 68.0 & 59.7 \\
$\quad$ Leche y crema & 54.0 & 50.8 & 49.9 & 38.0 & 26.5 & 23.7 \\
$\quad$ Mantequilla & 8.5 & 10.0 & 11.9 & 19.1 & 18.1 & 9.1 \\
$\quad$ Sueros & 1.8 & 1.4 & 2.1 & 3.2 & 4.0 & 9.7 \\
$\quad$ Queso & 11.6 & 13.3 & 7.9 & 12.1 & 11.2 & 8.7 \\
$\quad$ Caseína & 5.4 & 4.9 & 5.5 & 7.1 & 8.2 & 8.3 \\
Carne de ovino y bovino & 2.6 & 4.6 & 4.5 & 6.1 & 9.7 & 17.7 \\
Cueros y pieles & 3.0 & 2.3 & 2.0 & 1.5 & 1.2 & 0.3 \\
Frutas y vegetales & 0.1 & 0.2 & 0.2 & 0.4 & 0.8 & 0.7 \\
No agropecuarias & 13.0 & 12.5 & 16.0 & 12.5 & 20.3 & 14.5 \\
\hline
\end{tabular}

Fuente: Bancomext, World Trade Atlas, 2001.

Ministry of Foreign Affairs and Trade, External Trade Statistics, Nueva Zelanda, 2005. 
importaciones que realiza aquel país desde el nuestro han presentado bajos niveles de penetración en su mercado. En 2005 nuestro país ocupó el séptimo lugar como destino de las exportaciones neozelandesas de productos lácteos en general: en caseína ocupó el cuarto lugar; en mantequilla el quinto, en queso el octavo y en leche y crema el noveno puesto. De hecho, hoy en día las exportaciones de productos lácteos de Nueva Zelanda hacia México suman 60 por ciento de las exportaciones totales de aquel país hacia el nuestro y, si se agregan otros productos como carne, pieles y cueros, la participación de las exportaciones agroalimentarias se eleva a alrededor de 86 por ciento respecto del total (cuadro 5).

Por su parte, las exportaciones de México hacia Nueva Zelanda corresponden fundamentalmente a manufacturas, entre

\section{Cuadro 6}

Nueva Zelanda: importaciones desde México, 2003-2005

(estructura porcentual)

\begin{tabular}{lrrr}
\hline Productos & \multicolumn{1}{c}{2003} & \multicolumn{1}{c}{2004} & \multicolumn{1}{c}{2005} \\
\hline Total & 100.0 & 100.0 & 100.0 \\
Aparatos de transmisión & 13.2 & 12.4 & 16.2 \\
y componentes & & & \\
Computadoras & 6.0 & 6.2 & 11.6 \\
Circuitos integrados, & 7.8 & 7.4 & 7.6 \\
diodos y transistores & & & \\
Vehículos de motor & 6.8 & 11.3 & 7.1 \\
Cerveza & 2.7 & 2.8 & 5.9 \\
Alcohol etílico & 4.3 & 4.3 & 4.2 \\
Equipo telefónico & 6.1 & 6.9 & 3.9 \\
Maquinaria y partes & 14.0 & 7.5 & 3.9 \\
Ácidos saturados & 0.3 & 1.1 & 3.1 \\
Instrumentos médicos o & 1.1 & 2.9 & 2.7 \\
veterinarios & & & \\
Artículos de papel & 0.2 & 0.9 & 2.2 \\
Micrófonos y sus & 2.0 & 1.9 & 2.0 \\
accesorios & & & \\
Otros & 35.5 & 34.4 & 29.6 \\
\hline
\end{tabular}

Fuente: Ministry of Foreign Affairs and Trade, External Trade Statistics, Nueva Zelanda, 2005. las que sobresalen aparatos de radiocomunicación, computadoras, componentes para la industria electrónica, vehículos de motor y maquinaria, los cuales representan cerca de dos terceras partes del total exportado a aquel país, observándose un crecimiento medio anual de 17 por ciento durante el periodo 2000-2005. ${ }^{11}$ Los bienes agroalimentarios presentan una participación mucho menor, destacando aquí las bebidas alcohólicas con 10 por ciento del total, mismas que tienen una buena aceptación en el mercado neozelandés. Otros productos mexicanos que se están exportando a Nueva Zelanda son ácidos saturados, instrumentos médicos o veterinarios y artículos de papel (cuadro 6).

Este relativo crecimiento en el intercambio comercial entre los dos países responde a esfuerzos similares de ambos lados por ampliar sus relaciones con diversas economías. Para Nueva Zelanda, la mayor penetración de sus productos en el mercado mexicano constituye el primer paso de una bien definida estrategia para incursionar posteriormente en los demás mercados de Latinoamérica, principalmente. Si se toma en cuenta el fuerte potencial de exportación de productos lácteos y sus derivados que tiene Nueva Zelanda, por un lado, y la creciente demanda de los mismos en la mayoría de las economías en desarrollo en América, incluido México por supuesto, se puede formar una idea de las grandes posibilidades de aceptación de sus productos en dichos mercados.

Del lado de México, en los últimos años diversos sectores de la sociedad (el académico, el empresarial y hasta el gubernamental) han alzado su voz para insistir en la imperiosa necesidad que tiene nuestro país de estrechar más sus vínculos económicos con las diversas economías del Pacífico Asiático, en virtud del rápido 
crecimiento que han experimentado la mayoría de las mismas, como una forma de contrarrestar el abrumador peso que tiene la economía estadounidense sobre la mexicana. De ahí la importancia de incursionar más en los mercados asiáticos con objeto de diversificar nuestros mercados y aprovechar mejor las nuevas oportunidades que el crecimiento de aquellos países brinda a la economía mexicana.

Cabe subrayar que la neozelandesa y la mexicana son economías consideradas hasta cierto punto más complementarias que competidoras entre sí, ya que mientras el comercio externo de Nueva Zelanda se basa primordialmente en bienes agroalimentarios, el de México está constituido en su mayor parte por manufacturas.

Mediante negociaciones comerciales bilaterales y multilaterales, Nueva Zelanda ha pugnado por reducir los esquemas proteccionistas de otros países a fin de disminuir los fuertes obstáculos que enfrentan el comercio y la inversión a escala internacional, como son las barreras comerciales y no comerciales, aspectos de carácter técnico y otros relativos a la salud (la seguridad sanitaria y fitosanitaria), dada la fuerte dependencia de la economía neozelandesa respecto de las exportaciones agroalimentarias. En el caso de México, la diversificación de mercados tiene una alta prioridad en virtud de los riesgos de diversa índole que conlleva la fuerte dependencia de un solo mercado.

Por lo anterior, la eventual firma de un acuerdo comercial entre Nueva Zelanda y México, como lo han venido manifestando los neozelandeses, significaría para México una mayor participación en aquellos sectores poco competitivos de Nueva Zelanda como el textil, del vestido y del calzado, así como en otros en que nuestro país tiene mayores ventajas comparativas; tal es el caso de la maquinaria y equipo y de los electrónicos.
En el sector agropecuario, dado que los dos países se ubican en hemisferios opuestos, pueden ampliarse las oportunidades para ambas partes debido a la complementariedad estacional que presenta la producción agrícola para cada uno. Nueva Zelanda tiene ya una alta participación en las importaciones mexicanas de productos lácteos, pero existen otros rubros como las frutas y vegetales - cuya producción y competitividad está teniendo un fuerte crecimiento en aquel país-que podrían incursionar con éxito en nuestro país.

\section{La inversión extranjera directa (IED) de Nueva Zelanda en México}

Durante el periodo 1999-2005 México captó un total de 115 mil millones de dólares de inversión extranjera directa (IED). Por su origen, cerca de 80 mil millones provinieron de las economías integrantes del Mecanismo de Cooperación Económica Asia-Pacífico (APEC), lo que representa 69 por ciento del total y en donde Estados Unidos aporta un 63 por ciento. Por su parte, las economías del Pacífico Asiático registran una IED acumulada de 3.6 mil millones de dólares en el mismo periodo, lo cual representa 3 por ciento del total. Por orden de importancia están Japón, Singapur, Corea del Sur, Taiwán, Chile, Nueva Zelanda, China, Australia y Hong Kong. ${ }^{12}$

En el mismo periodo - 1999-2005- la IED acumulada de Nueva Zelanda en México sumó cerca de 74 millones de dólares, cantidad menor a 1 por ciento del total captado por este último. Cabe anotar que casi la totalidad de este capital arribó a nuestro país en los últimos cinco años, por lo que antes del año 2000 la IED neozelandesa en México era prácticamente nula. De igual forma, es importante anotar que del total de la inversión de Nueva Zelanda en territorio mexicano, 
alrededor de 72 por ciento se ha orientado al sector de manufactura, particularmente a la elaboración de productos lácteos; el resto se ha dirigido al sector servicios. En cuanto a su distribución geográfica, 96 por ciento de la IED neozelandesa está concentrada en el Distrito Federal, y el resto se ubica principalmente en Jalisco y Guanajuato (cuadro 7).

En cuanto a las empresas neozelandesas que han invertido en México, Fonterra es la más importante. Al ser la empresa de productos lácteos más grande de Nueva
Zelanda ha procurado establecer alianzas con empresas mexicanas en ese sector para incursionar en el mercado mexicano; para ello estableció importantes vínculos con Liconsa y recientemente adquirió tres empresas procesadoras de queso en nuestro país: La Mesa, Eugenia y Noche Buena; además, recientemente abrió un centro de investigación agropecuario en Jalisco. Las ventas de Fonterra en México ascienden actualmente a 200 millones de dólares por año. La segunda empresa neozelandesa de importancia en México es Tru-test, la

\section{Cuadro 7}

Nueva Zelanda: distribución de la inversión neozelandesa en México, 1999-2005 (miles de dólares)

\begin{tabular}{lrrrrrrrrr}
\hline & 1999 & 2000 & 2001 & 2002 & 2003 & 2004 & $2005^{*}$ & $1999-2005^{*}$ & $\%$ \\
\hline Sectores & & & & & & & & & \\
\hline Total & 38.5 & 24.9 & $1,655.4$ & $62,330.9$ & $6,514.7$ & $3,025.7$ & 116.5 & $73,706.8$ & 100.0 \\
Agropecuario & 0.0 & 0.0 & 0.0 & 0.0 & 0.0 & 0.0 & 0.0 & 0.0 & 0.0 \\
Minería y extracción & 0.0 & 0.0 & 0.0 & 0.0 & 23.9 & -21.4 & 0.0 & 2.5 & 0.0 \\
Manufactura & 0.0 & 0.0 & 789.7 & $43,070.5$ & $6,260.5$ & $2,652.0$ & 84.8 & $52,857.5$ & 71.7 \\
Electricidad y agua & 0.0 & 0.0 & 0.0 & 0.0 & 0.0 & 0.0 & 0.0 & 0.0 & 0.0 \\
Construcción & 0.0 & 0.0 & 17.0 & 0.0 & 0.0 & 0.0 & 0.0 & 17.0 & 0.0 \\
Comercio & 0.0 & 5.3 & 3.1 & 12.1 & 2.4 & -2.3 & 0.0 & 20.5 & 0.0 \\
Transportes y & 0.0 & 0.0 & 0.0 & 0.3 & 0.0 & 0.0 & 0.0 & 0.3 & 0.0 \\
comunicaciones & & & & & & & & & \\
Servicios financieros & 0.0 & 0.0 & 27.1 & 1.6 & 0.0 & 0.0 & 27.1 & 55.9 & 0.1 \\
Otros servicios & 38.5 & 19.6 & 818.5 & $19,246.5$ & 228.0 & 397.3 & 4.6 & $20,753.1$ & 28.2 \\
\hline Entidad federativa & & & & & & & & & \\
\hline Total & 38.5 & 24.9 & $1,655.4$ & $62,330.9$ & $6,514.7$ & $3,025.7$ & 116.5 & $73,706.8$ & 100.0 \\
Distrito Federal & 35.5 & 0.0 & 55.1 & $62,292.6$ & $5,668.8$ & $2,633.3$ & 4.6 & $70,690.0$ & 95.9 \\
Jalisco & 0.0 & 5.3 & 807.5 & 35.0 & 188.8 & 395.6 & 0.0 & $1,432.2$ & 1.9 \\
Guanajuato & 0.0 & 0.0 & 789.7 & 0.0 & 589.4 & 0.0 & 0.0 & $1,379.1$ & 1.9 \\
Baja California & 0.0 & 0.0 & 0.0 & 0.9 & 4.6 & 17.7 & 84.8 & 108.1 & 0.1 \\
Yucatán & 0.0 & 0.0 & 0.0 & 0.0 & 24.0 & 0.0 & 27.0 & 51.0 & 0.1 \\
Baja California Sur & 0.0 & 19.6 & 0.0 & 2.4 & 0.0 & 0.0 & 0.0 & 22.0 & 0.0 \\
Nayarit & 0.0 & 0.0 & 3.1 & 0.0 & 15.2 & 0.0 & 0.0 & 18.3 & 0.0 \\
Qintana Roo & 3.0 & 0.0 & 0.0 & 0.0 & 0.0 & 0.4 & 0.0 & 3.5 & 0.0 \\
Chihuahua & 0.0 & 0.0 & 0.0 & 0.0 & 23.9 & -21.4 & 0.0 & 2.5 & 0.0 \\
Guerrero & 0.0 & 0.0 & 0.0 & 0.0 & 0.0 & 0.0 & 0.1 & 0.1 & 0.0 \\
\hline
\end{tabular}

* Enero-septiembre de 2005.

Fuente: Secretaría de Economía, Dirección General de Inversión Extranjera, 2005. 
cual fabrica cercas electrificadas para la protección del ganado y cuenta con instalaciones en las ciudades de Monterrey y Tampico. ${ }^{13}$

De igual forma, Nueva Zelanda ha manifestado un creciente interés por invertir en otras industrias de nuestro país, particularmente en la del vestido y de tapetes, con la intención de confeccionarlos y comercializarlos en México, así como de exportarlos a otros mercados aprovechando la extensa red de acuerdos de libre comercio que México ha signado con diversos países de América, Europa y Asia.

Por el contrario, las oportunidades de inversión que se le presentan a México en Nueva Zelanda se encuentran principalmente en los sectores de tecnologías de la información y de comunicaciones, biotecnología, manufacturas especializadas, procesamiento de madera y producción cinematográfica, dado el creciente auge de la industria del cine y la televisión en aquel país. También la horticultura, la industria forestal y de procesamiento y comercio de alimentos ofrecen interesantes oportunidades de negocios para los inversionistas mexicanos.

\section{Conclusiones}

Nueva Zelanda cuenta con una larga tradición en el comercio internacional gracias a una favorable dotación de factores que han hecho posible desarrollar un fuerte y competitivo sector exportador agropecuario. Esta vocación se inició desde mediados del siglo XIX, cuando formalizó su integración con Gran Bretaña y rápidamente se convirtió en un importante proveedor - principalmente de carne de ovino, productos lácteos y lana- de ése y de otros países de Europa. No obstante, en la segunda mitad del siglo xx la combinación de diversos sucesos tornó desfavorables los términos de intercambio, que afectaron drásticamente a Nueva Zelanda, ya que las exportaciones agropecuarias se habían convertido en la base de su economía; entre estos sucesos estaban el paulatino deterioro de los precios de los bienes agropecuarios en el mercado internacional, la integración de Gran Bretaña a la Comunidad Económica Europea (hoy Unión Europea) y el fortalecimiento de los esquemas proteccionistas en la agricultura a escala mundial.

Por lo tanto, Nueva Zelanda se vio obligada a emprender una nueva estrategia comercial basada, por un lado, en la diversificación de sus relaciones comerciales principalmente hacia los mercados del Pacífico Asiático, por su cercanía geográfica y por el rápido crecimiento económico mostrado por las economías de la región y, por otro, mediante la ampliación de su base de exportaciones, al incorporar a sus tradicionales exportaciones de carne, lácteos y lana, otros bienes como productos forestales y pesqueros, frutas y vegetales y manufacturas diversas. Al mismo tiempo, Nueva Zelanda se convirtió en un activo promotor de la liberalización del comercio agropecuario en los foros mundiales y regionales, por los evidentes beneficios que ello representa para su economía.

Como parte de esta nueva política comercial, Nueva Zelanda ha emprendido importantes esfuerzos para ampliar sus relaciones comerciales con México y, con ello, incursionar en el mercado mexicano y en prácticamente todos los países del continente americano, aprovechando los estratégicos acuerdos comerciales que nuestro país tiene en gran parte del mundo. Para México, la relación bilateral con Nueva Zelanda también representa otra oportunidad más para diversificar su comercio hacia aquella región del mundo y disminuir la fuerte de- 
pendencia que tiene respecto del mercado estadounidense.

El hecho de que la economía y el mercado de Nueva Zelanda sean relativamente pequeños, no significa que no existan oportunidades de negocios para México. Con base en una nueva política comercial de largo plazo y con una bien diseñada estrategia de vinculación con las economías asiáticas, se puede incursionar no sólo en el mercado de Nueva Zelanda sino en otros de la región como Australia, por la cercanía geográfica y la fuerte integración económica que ambas naciones han desarrollado. niv

\section{Notas}

1 Con base en Far Eastern Economic Review, abril 13 de 2000.

2 Véase Ministry of Agriculture and Forestry, s/f.

3 Ídem.

4 Véase CEPAL, 1993, p. 31.

5 Ídem.

6 Véase APEC, 2000, p. 11.

7 Con base en FMI, 2005.

8 Con base en Ministry of Foreign Affairs and Trade, 2005.

9 FMI, op. cit.

10 Con base en Bancomext, s/f.

11 Con base en Ministry of Foreign Affaire and Trade, op. cit., 2005.

12 Con base en Secretaría de Economía, 2005.

13 Con base en información de Felipe Gazcón, El Financiero, 14 de julio de 2003.

\section{Bibliografía}

APEC (2000) New Zealand's 2000 APEC Individual Action Plan, Bandar Seri Begawan, noviembre.

Bancomext (s/f) Apéndice estadístico. http://www. bancomext.gob.mx

— (2001) World Trade Atlas, 2001.

CEPAL (1993) El proceso exportador en tres agriculturas templadas del Hemisferio Sur (Australia, Nueva Zelanda y Sudáfrica), División de Desarrollo Productivo y Empresarial, documento LC/R 1350, 30 de diciembre, Santiago de Chile.

Evans, Lewis, Arthur Grimes, y Bryce Wilkinson (1996) "Economic Reform in New Zealand 1984-1995: The Pursuit of Efficiency", Journal of Economic Literature, vol. XxxIV, diciembre.

Far Eastern Economic Review (2000), abril 13.

Fondo Monetario Internacional (FMI) (2005) International Financial Statistics, vol. LVIII, núm. 7, julio, Washington, D. C.

Ministry of Agriculture and Forestry (s/f) New Zealand: A Land of Contrasts. http://www.maf.govt. $\mathrm{nz} / \mathrm{MAFnet}$.

Ministry of Foreign Affairs and Trade (2005) External Trade Statistics, Nueva Zelanda.

OCDE (2002) OECD EConomic Surveys. New Zealand, vol. 2002, Francia.

Rae, Allan (1996) Agricultural can Survive Unilateral Reforms: Lessons from Down Under, Conference on Food and Agricultural Policy Challengers for the Asia-Pacific, Manila, Filipinas, 1-3 de octubre.

Sandrey, Ron A., y M. Scobie Grant (1994) "Changing International Competitiveness and trade: Recent Experience in New Zealand Agriculture", American Journal of Agricultural Economics, vol. 76, núm. 5, diciembre. Estados Unidos: American Economic Association.

Secretaría de Economía (2005) Dirección General de Inversión Extranjera, México. 